\title{
Multi-panel, on-single-chip Memristive Biosensing
}

\author{
Ioulia Tzouvadaki, Abuduwaili Tuoheti, Séverine Lorrain, Manfredo Quadroni, Marie-Agnès Doucey, \\ Giovanni De Micheli, Danilo Demarchi, Sandro Carrara
}

\begin{abstract}
Memristive biosensors have demonstrated excellent capabilities for ultrasensitive bio-detection. In the present work, memristive biosensing chips are designed, fabricated and implemented in a for the first time presented multi-panel on-chip detection for discrete sensing of a target molecule through separate functionalization of individual devices on the same chip. The biosensing scheme is validated by means of labeled (i.e. fluorescence) and label-free (i.e. electrical) characterization methods. This novel memristive multi-panel sensing paradigm paves the way for fast and ultrasensitive PoC (point-of-care) devices allowing the detection of specific targets in complex matrixes where non-specific molecules are present as well, and opening great potential for the application of memristive phenomena in multiplexed ultrasensitive bio-detection and theranostics.
\end{abstract}

Index Terms - Memristive Biosensors; Bio-functionalization; Multi-panel detection;

\section{INTRODUCTION}

$\mathrm{S}$ EMICONDUCTOR nanowires may provide low-cost microchips and therefore are considered as effective and highly promising building blocks for miniaturized bioassays dedicated to medical applications, in both diagnostics and therapeutics. In this framework, silicon nanowire-arrays that exhibit memristive electrical properties [1]-[3] are biofunctionalized with receptor molecules i.e. antibodies or DNA aptamers, giving rise to the so-called memristive biosensors. These memristive sensors have already successfully achieved ultrasensitive detection for cancer biomarkers, demonstrating atto-molar concentration sensing performance [4] as well as effective screening of therapeutic compounds, along with the possibility for continuous drug monitoring [5] therefore showing immense potential for ultrasensitive and precise biosensing. The bio-detection is based on the hysteresis

Financial support received from the multidisciplinary FNS Grant CR32I3 156915.

Ioulia Tzouvadaki, Giovanni de Micheli and Sandro Carrara are with the Integrated System Laboratory, EPFL, Lausanne, Switzerland (e-mail: ioulia.tzouvadaki@alumni.epfl.ch,giovanni.demicheli@epfl.ch, sandro.carrara@epfl.ch )

Abuduwaili Tuoheti and Danilo Demarchi are with Department of Electronics and Telecommunications, Politecnico di Torino, Torino, Italy (e-mail: abuduwaili.tuoheti@polito.it, danilo.demarchi@polito.it )

Séverine Lorrain and Manfredo Quadroni are with the university of Lausanne Switzerland (UNIL) (e-mail: severine.lorrain@unil.ch, manfredo.quadroni@unil.ch )

Marie-Agnès Doucey is with the university of Lausanne Switzerland (UNIL) and the Ludwig Institute for Cancer Research, Lausanne, Switzerland (e-mail: marie-agnes.doucey@unil.ch ). modification appearing in the electrical characteristics upon the introduction of charged biological species on the nanodevices' surface (bio-functionalization procedures and target molecule uptake) [6], [7], [8].

Moreover, the design and realization of memristive biosensing electronic platforms was realized and validated [9], [10] providing a fast, fully-automatized and simultaneous sensing output of multiple individual memristive biosensors on a single chip. Highlights of the development steps of memristive biosensors are summarized in Table I.

Table I. Developing Memristive Biosensors

\begin{tabular}{|l|l|}
\hline \multicolumn{2}{|l|}{ Physical and Chemical Sensing } \\
\hline Anti-rabbit & {$[6]$} \\
\hline $\mathrm{pH}$ & {$[11]$} \\
\hline VGEF & {$[12]$} \\
\hline Prostate Specific Antigen (PSA) & {$[4]$} \\
\hline Tenofovir (TFV) & {$[5]$} \\
\hline Theoretical Study and Modelling \\
\hline Equivalent circuit modeling & {$[7],[13]$} \\
\hline Physical modeling & {$[8]$} \\
\hline Integration, optimization towards PoC schemes \\
\hline Bio-functionalization & {$[14]$} \\
\hline Microfluidics & {$[15]$} \\
\hline Electronic platforms & {$[9][10]$} \\
\hline Multi-panel on-chip sensing & Present work \\
\hline
\end{tabular}

Meanwhile, the importance of multi-panel detection in a labon-a-chip framework is even more pronounced when considering the aspect of theranostics applications where both disease biomarkers and therapeutic compounds are simultaneously monitored in order to reflect the effect of the treatment [16]-[19]. This scheme may involve target molecules that are competing or complementary to each other. The first case concerns for instance a disease marker and the specific therapeutic drug or its metabolites, evaluating the efficiency of the medical treatment in the form of a feedback loop, allowing appropriate adjustments for enhancing the therapy [20]-[22]. The case of complementary markers refers to simultaneous detection of multiple biomarkers indicating the same disease or disorder targeting at a more accurate evaluation of the disease evolution and providing enhanced diagnosis [23]. Furthermore, the discrete sensing of specific biomarkers when included in a more complex matrix or cocktail of multiple substances consists another significant challenge in biosensing.

In this work, a proof-of-concept study demonstrating multipanel detection paradigm involving memristive biosensors and individualized sensing is for the first time presented and validated through electrical and fluorescence characterization techniques. This biosensing scheme paves the way for 
individualized biomarker sensing in a complex matrix, as well as for multiple biomarkers/drugs detection on the same chip and application in theranostics. Sensing chips of memristive biosensors conjugated with metallic extension electrodes are validated for successful sensing performance and then implemented for multi-panel sensing.

\section{MATERIALS AND METHODS}

\section{A. Memristive Biosensing chips}

Two-terminal silicon ( $\mathrm{Si}$ ) nanofabricated wire-arrays exhibiting memristive electrical properties are acquired through a top-down fabrication process using Silicon-on-Insulator (SOI) wafers as described in [7], by implementing electron beam lithography and Deep Reactive Ion Etching (DRIE). The wires are suspended and anchored between Nickel Silicide (NiSi) pads forming Schottky-barriers and serving for the electrical characterization of the nanodevices. Metal lines are integrated on top of the already fabricated nanostructures and serve as extension line electrodes to the NiSi pads of the devices, as described in [9]. The metal lines are designed taking into consideration the fact that the hysteretic properties of the memristive biosensors are sensitive to additional resistive elements introduced in series to the nanofabricated devices, eliminating or even completing masking the memristive properties of the nanodevices. For this reason, the extension lines design follows the outcomes of a relevant computational study and calculations [9], [13] for respecting the resistance tolerance of the system. A common source and separated drains are considered for individual measurement of twelve integrated sensors (as seen in Fig. 1) that allow possibility for adequate statistical analysis to determine average value and standard deviation. This statistical analysis is required for obtaining reliable results and overcome the variability of the sensors originating from the DRIE process. In addition, such realization of multiple sensors allows the possibility for simultaneous sensing (multi-panel sensing), that consists the main aim of the present study.

To confirm the sound functionality of the metal lines the memristive devices were functionalized by exposure of the surface to antibody against Prostate Specific Antigen (PSA), i.e. anti-PSA antibody (Abcam-ab10185), solution in Phosphate Buffered Saline (PBS) (pH 7.4 Sigma-Aldrich) for $4 \mathrm{~h}$ at room temperature, thoroughly rinsed with the same buffer and gently dried with $\mathrm{N}_{2}$ flow. Then, the antigen uptake was performed through successive $45 \mathrm{~min}$ incubations of the sensing chip in solutions of PSA (Millipore Angebot R-1939458.1; 539834 purchased from Merck) in PBS. The measurements were performed with the prober needles placed on the Pt pads instead of the NiSi pads.

The electrical monitoring of the memristive nano-bio-sensors was performed utilizing the configuration consisting of a Cascade Microtech Probe Station in combination to a HewlettPackard 4165A Precision Semiconductor Parameter Analyzer. Fluorescence characterization is carried out using scanner Typhoon Trio (GE Healthcare).

\section{B. Multiplexing approach for sensing}

A single-chip, detection in a multi-panel mode is for the first time attempted implementing memristive biosensors. The memristive devices included in the same $1 \mathrm{~cm} \mathrm{x} 1 \mathrm{~cm}$ chip are first subjected to a silanization step with GPTES (440167, Sigma-Aldrich). Then the devices are separately biofunctionalized with different antibody solutions. Antibodies either from mouse, goat or rabbit were diluted within PBS to get a final concentration of $0.5 \mathrm{mg} / \mathrm{ml}$ with $0.5 \%$ trehalose (T9449, D-(+)-Trehalose dihydrate from corn starch $=99 \%$, Sigma). Multiplexed biosensing requires post-fabrication nanowire functionalization with probe molecules, (though robotic spotting [24], ink-jet printing [25], selective heating [26], electrochemically [27], or via dip-pen nanolithography [28]). In this work, a micro-spotter (GeSim NanoPlotter 2.1) is used for specific and separate bio-functionalization of each nanodevice with the different antibodies under consideration, with a drop of $400 \mathrm{pL}$ antibody-solution of an initial diameter set at $150 \mu \mathrm{m}$. A rabbit antibody is used as a positive-control bio-functionalization reagent and is spotted to various devices of the sensing chip. Meanwhile, three different negative controls are taken into consideration: a mouse and a goat antibody both applied at the same concentrations as the positive control as well as plain buffer solution (PBS).

The micro-spotting procedure finally results in discrete biofunctionalization of different devices (Fig.1). Following the bio-functionalization the chip remains in a humid chamber for $1 \mathrm{~h}$ in order to ensure the attachment of the bio-functionalization regents on the nanodevice surface. The additional humidity introduced, aims at the conservation of the liquid antibodysolution drops which are very sensitive to evaporation issues. In order to prevent non-specific binding of proteins during the detection measurement, the remaining active GPTES-derived groups are passivated by applying $10 \mathrm{mM}$ ethanolamine in PBS solution at room temperature. Washing process is further performed to remove unreacted molecules.

An additional blocking step is carried out with PBS containing $3 \%$ gelatin from cold water fish skin. This further blocking forms a stable and specific receptor layer, due to the passivation property of neutrally charged gelatin molecules that aims to prevent the empty sites of the device active area from interacting with charged non-specific species from the analyte solution. Then, what follows is the antigen uptake through simultaneous incubation of all the nanodevices under

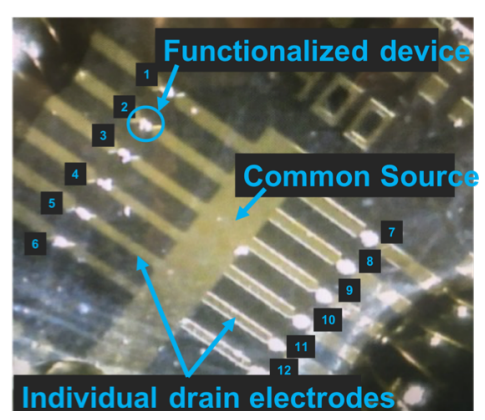

Fig. 1. Optical microscopy image depicting the selective spotting with receptor molecules. A closer view of the surface of the multi-sensing chip is shown, depicting the twelve sensors locations, that are integrated with metal lines. The discrete functionalization scheme is visible for each individual sensor and shown as a droplet located exactly at the sensor's regions. The memristive devices are integrated with the extension $\mathrm{Pt}$ electrodes in order to enable the electrical characterization as well as the integration with the electronic readout platform as reported in [9] and [10]. 
consideration for 45 min in humid environment. A pipette of 2 $\mu \mathrm{L}$ is used to efficiently transfer a sufficient amount of antigen solution directly and restricted at the region of interest i.e. nanowires' region. Two different aspects are considered through the antigen uptake. A selected region of the chip is incubated in a high concentration of antigen-tagged with fluorescent substance, destined for a fluorescence study and another region is incubated with successive $45 \mathrm{~min}$ incubations increasing antigen concentrations belonging to the range close and below the clinical range of most biomarkers, like for example PSA. Each incubation is followed by a washing step with gelatin, two washing steps with PBS and a final washing step with PBS $10 \%$ in order to remove any salts originated from the buffer.

\section{RESULTS AND DISCUSSION}

\section{A. Performance of Memristive Biosensing chips}

The electrical response using the fabricated chips is acquired before and after the bio-functionalization process and antigen uptake with increasing antigen concentration (Fig. 2). The obtained results clearly show that the hysteresis is successfully maintained and the voltage gap is acquired as expected upon bio-functionalization and decreases with the increasing antigen uptake as shown in Fig. 2.

\section{B. Multi-panel sensing with memristive biosensors}

For the multi-panel detection two separate methods (fluorescence detection and label-free sensing through electrical characterization) are independently implemented to verify the multi-panel sensing capabilities of the memristive biosensors.

\section{1) Fluorescence Sensing}

The results obtained from fluorescence characterization are illustrated in Fig. 3. It is depicted that selected biofunctionalization is successfully achieved and only the devices functionalized with the positive control antibody finally demonstrate the capability to bind the target antigens. Despite the fact that accurate bio-functionalization is achieved strictly

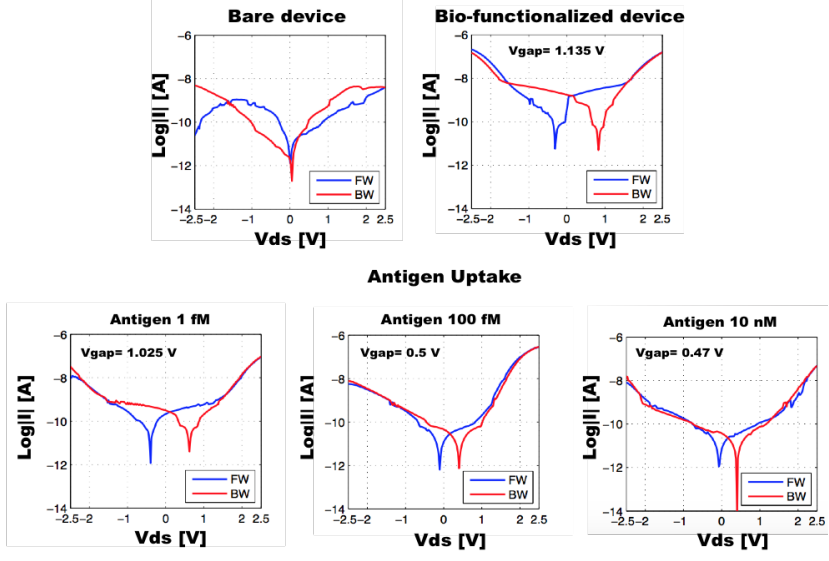

Fig. 2. Indicative semi-logarithmic current to voltage characteristics obtained for the same device measured at the Pt pads using the Probe Station and Keithley configuration. The different graphs correspond to the bare device, after the bio-functionalization with $\mathrm{Ab}$ and with increasing antigen uptake (from $1 \mathrm{fM}$ to $10 \mathrm{nM}$ ).

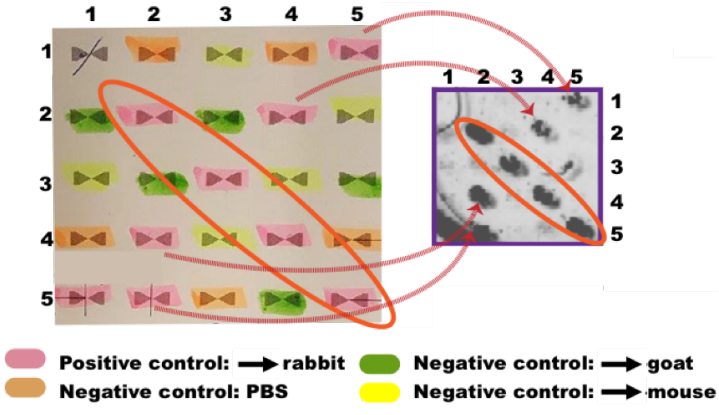

Fig. 3. Fluorescence results demonstrate the successful multi-panel biofunctionalization.

at the region of each suspended nanowire, due to adhesion phenomena occurring during the incubation time the biofunctionalization solution is slightly spread outside the target area and finally stabilized to a slightly larger droplet, after established hydrodynamic and hydrostatic forces equilibrium, as well as evaporation aspects.

\section{2) Label-free sensing}

The nanodevices, bio-functionalized with the different antibodies and the PBS, are electrically characterized (baseline measurement), followed by the electrical monitoring of the nanodevices with increasing antigen concentrations. The introduction of charged residues on the surface of the nanodevices, as it is expected, induces the appearance of a voltage gap in the semi-logarithmic current to voltage characteristics. Meanwhile, the binding of antigen (through the receptor-target molecule relation) introduces a masking contribution to the effect already brought by antibodies, resulting in a decreasing voltage gap. Indeed, the voltage gap presented at the electrical characteristics of the nanodevices functionalized with the positive control antibody demonstrates smaller values after the antigen uptake, verifying successful antibody-antigen binding (Fig. 4). However, no significant difference is recorder for the case of the negative-control biofunctionalized devices (Fig. 4). Further study of the analytical

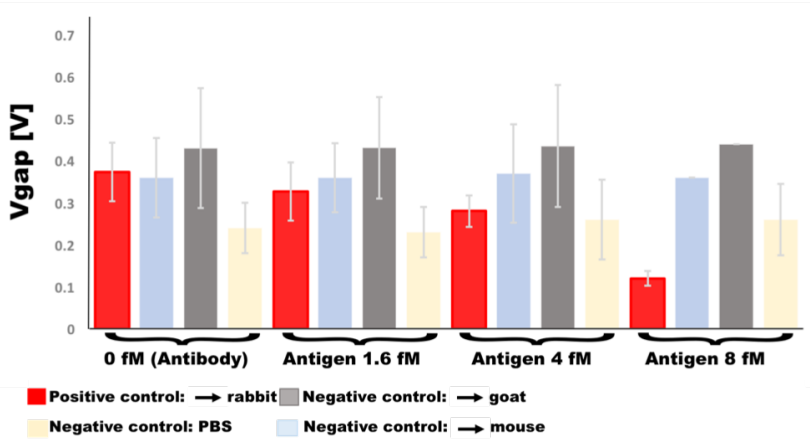

Fig. 4. Electrical characterization results bringing proof of the successful multipanel bio-functionalization. The average value of the voltage gap acquired for increasing concentrations of four different molecules which are the target molecule and three negative controls. No significant signal difference is depicted for the case of the negative-control bio-functionalized sensors in contrast to the decreasing voltage gap trend with increasing concentration shown for the case of the target molecule. 


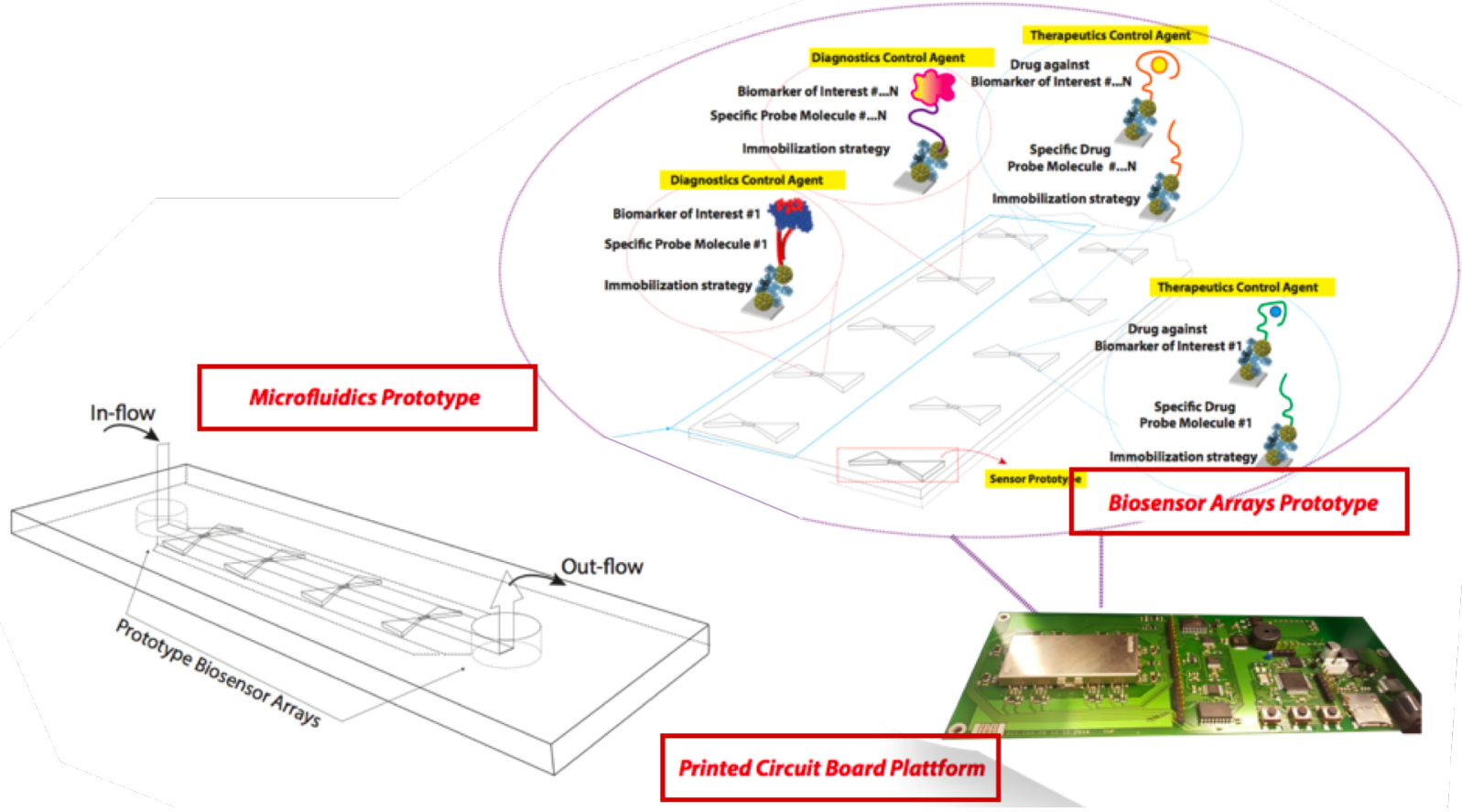

Fig. 5. Illustration of the memristive bio-sensing platform, implemented for theranostics in combination with specially designed microfluidic circuit.

performance of the nanodevices at the different antigen steps, demonstrates a decreasing behavior only for the nanodevices functionalized with the positive control antibody while the voltage gap for the other nanodevices remains constant at the levels of the values after the bio-functionalization. This finding brings proof of the possibility for efficient individualized biofunctionalization of different nanodevices on the same chip. Moreover, these results demonstrate the capability of the memristive biosensors to provide discrete sensing of a target molecule against other non-specific molecules through a tailored functionalization of individual devices. Therefore, this study consists a very important step towards future implementation of the memristive biosensors for the detection of a specific biomarker or drug when it is included in a cocktail of substances where other (non-specific) biomarkers or drugs are included as well. As a more extended version of this scheme, multiple biomarkers can be detected paving the way for multi-panel applications and simultaneous bio-detection of different biomarkers on the same chip. Having proven the multi-panel memristive bio-sensing paradigm, this scheme can be combined in a straight-forward way with the memristive biosensing board prototype [10] as well as with the microfluidic circuits especially designed for memristive biosensors [15], as illustrated in the complete scheme shown in Fig. 5.

\section{CONCLUSIONS}

In this work, a proof-of-concept multi-panel on-single-chip detection scheme implementing memristive biosensors is for the first time presented and validated. First, specially designed memristive sensing chips offering disposable sensing modules by integrating memristive wires and extension metal electrodes are validated for successfully providing the electrical characteristics of the devices before and after the biofunctionalization and the target uptake steps. Most importantly, this novel sensing paradigm demonstrates successful implementation of the memristive biosensors in a multi-panel sensing scheme in both labeled and label-free sensing. The system presented in this work paves the way for advanced biodetection with memristive biosensors, allowing individualized detection of specific targets in complex matrixes in the presence of non-specific molecules or in even more complex schemes i.e. cells, tumor extracts, holding also great promise for fast and ultrasensitive sensing in theranostics.

\section{REFERENCES}

[1]L. Chua, "Memristor-The missing circuit element" IEEE Trans. Circuit Theory, 18(5):507 - 519, 1971.

[2]L. Chua, S. Kang, "Memristive Devices and systems", Proc. IEEE, 64(2):209-223, 1976.

[3]L. Chua, "Resistance switching memories are memristors" Appl. Phys. A, 102(4):765-783, 2011

[4]I. Tzouvadaki and P. Jolly, X. Lu, S. Ingebrandt, G. de Micheli, P. Estrela and S. Carrara, "Label-Free Ultrasensitive Memristive Aptasensor" Nano Lett. 16: 4472-4476, 2016.

[5]I. Tzouvadaki and N. Aliakbarinodehi, G. De Micheli, S. Carrara, "The memristive effect as a novelty in drug monitoring" Nanoscale, 9, 9676 - 9684, 2017.

[6]S. Carrara, D. Sacchetto, M.A. Doucey, C. Baj-Rossi, G. De Micheli and Y. Leblebici, "Memristive-biosensors: A new detection method by using nanofabricated memristors", Sensor Actuat. B-Chem. 171-172:449-457, 2012.

[7]I. Tzouvadaki, F. Puppo, M.A. Doucey, G. De Micheli, S Carrara, "Computational Study on the Electrical Behavior of Silicon Nanowire Memristive Biosensors", IEEE Sensors Journal, 15(11), 6208-6217, 2015.

[8]F. Puppo, F.L. Traversa, M. Di Ventra, G. De Micheli, S. Carrara, "Surface trap mediated electronic transport in biofunctionalized silicon nanowires", Nanotechnology, 27, 345503, 2016.

[9]S. Naus and I. Tzouvadaki, P-E. Gaillardon, A. Biscontini, G. De Micheli, S. Carrara, "An Efficient Electronic Measurement Interface for Memristive 
Biosensors" IEEE International Symposium on Circuits and Systems (ISCAS) 2017.

[10] Ioulia Tzouvadaki, Abuduwaili Tuoheti, Giovanni De Micheli, Danilo Demarchi, Sandro Carrara, "Portable Memristive Biosensing System as Effective Point-of-Care Device for Cancer Diagnostics" IEEE International Symposium on Circuits and Systems (ISCAS) 2018.

[11] F. Puppo, M. Di Ventra, G. De Micheli, S. Carrara, "Memristive sensors for $\mathrm{pH}$ measure in dry conditions" Surf. Sci., 624, 76-79, 2014.

[12] F. Puppo, M.A. Doucey, M. Di Ventra, G. De Micheli, S. Carrara, "Memristor-Based Devices for Sensing" IEEE International Symposium on Circuits and Systems (ISCAS) 2014.

[13] I. Tzouvadaki, A. Vallero, F. Puppo, G. De Micheli, S. Carrara, "Resistance impact by long connections on electrical behavior of integrated Memristive Biosensors" IEEE International Symposium on Circuits and Systems (ISCAS) 2016.

[14] I. Tzouvadaki, N. Madaboosi, I. Taurino, V. Chu, J. P. Conde, G. De Micheli, S. Carrara, "Study on the bio-functionalization of memristive nanowires for optimum memristive biosensors" J. Mater. Chem. B ,4, 2153 2162, 2016.

[15] A. Vallero, I. Tzouvadaki, F. Puppo, MA. Doucey, J.F. Delaloye, G. De Micheli, S. Carrara, "Memristive Biosensors Integration with Microfluidic Platform" IEEE Trans. Circuits Syst. I, Reg. Papers 63(12), 21.

[16] C. Yang, C. Xu, X. Wang, X. Hu, "Quantum-dot-based biosensor for simultaneous detection of biomarker and therapeutic drug: first steps toward an assay for quantitative pharmacology" Analyst, 137, 1205-1209, 2012.

[17] S. Carrara, A. Cavallini, V. Erokhin, G. De Micheli, "Multi-panel drugs detection in human serum for personalized therapy" Biosensors and Bioelectronics 26, 3914-3919, 2011.

[18] M. Lee, KY. Baik, M. Noah, YK. Kwon, JO. Lee, S. Hong, "Nanowire and nanotube transistors for lab-on-a-chip applications" Lab Chip 9(16), 2267$80,2009$.

[19] YI. Zhang, R. Chen, L. Xu, Y. Ning, S. Xie, GJ. Zhang, "Silicon nanowire biosensor for highly sensitive and multiplexed detection of oral squamous cell carcinoma biomarkers in saliva" Anal Sci. 31(2), 73-8, 2015.

[20] M. Khairy, B. Mahmoud, C. Banks, "Simultaneous determination of codeine and its co-formulated drugs acetaminophen and caffeine by utilising cerium oxide nanoparticles modified screen-printed electrodes" Sens Actuators B Chem. 259, 142-154, 2018.

[21] M. Khairy, A. Khorshed, F. Rashwan, G. Salah, H. Abdel-Wadood, C. Banks, "Simultaneous voltammetric determination of antihypertensive drugs nifedipine and atenolol utilizing $\mathrm{MgO}$ nanoplatelet modified screen-printed electrodes in pharmaceuticals and human fluids" Sens Actuators B Chem. 252, 1045-1054, 2017.

[22] P. Manikandan, V. Dharuman, "Electrochemical Simultaneous Sensing of Melatonin, Dopamine and Acetaminophen at Platinum Doped and Decorated Alpha Iron Oxide" Electroanalysis 29(6), 1524-1531,2017.

[23] C. Wang, Z. Sun, L. Ma, M. Su, "Simultaneous Detection of Multiple Biomarkers with over Three Orders of Concentration Difference Using Phase Change Nanoparticles" Anal. Chem., 83(6),2215-2219, 2011.

[24] R. Auburn, D. Kreil, L. Meadows, B. Fischer, S. Matilla, S. Russell, "Robotic spotting of cDNA and oligonucleotide microarrays" Trends Biotechnol. 23, 374-379, 2005.

[25] A. Bietsch, J. Zhang, M. Hegner, H. Lang, C. Gerber, "Rapid functionalization of cantilever array sensors by inkjet printing" Nanotechnology, 15, 873-880, 2004.

[26] I. Park, Z. Li, A. Pisano, R. Williams, "Selective surface functionalization of silicon nanowires via nanoscale joule heating" Nano Lett. 7, 3106-3111, 2007.

[27] Y. Bunimovich, G. Ge, K. Beverly, R. Ries, L. Hood, J. Heath, "Electrochemically programmed, spatially selective bio-functionalization of silicon wires" Langmuir, 20,10630-10638, 2004.

[28] K. Salaita, Y. Wang, C. Mirkin, "Applications of dip-pen nanolithography" Nat Nanotechnol. 2, 145-155, 2007.

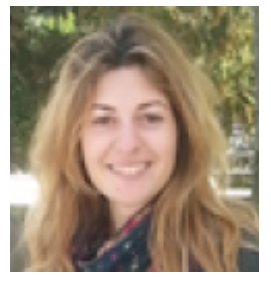

Ioulia Tzouvadaki received her B.Sc. degree in physics, from National and Kapodistrian University of Athens (U.O.A), Greece, the M.Sc. degree in microsystems and nanodevices from National Technical University of Athens (N.T.U.A) and the doctorate degree from EPFL, Switzerland. During her postgraduate studies, she worked at the Clean Room Laboratory of the National Center for Scientific Research (NCSR) Demokritos in the field of experimental construction processes concerning integrated circuits and the experimental characterization process of nanomaterials and nanodevices. Her M.Sc. thesis concerned the computational study and simulation of polymer nanocomposite materials, within the Computational Materials Science and Engineering (CoMSE) research group, of the School of Chemical Engineering at the NTUA. Her Ph.D. work at the Integrated System Laboratory (LSI) of EPFL, focused on the fabrication and characterization of nanostructures and their implementation as ultrasensitive nano-bio-sensors in both diagnostics and therapeutics.

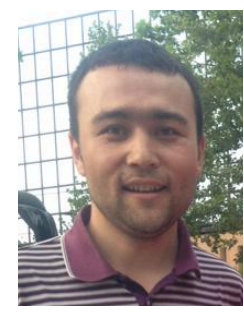

Abuduwaili Tuoheti was born in Xinjiang, China in 1984. He received B.Sc. Degree in Microelectronic Physics from Nanjing University (China) in 2008. Then he worked as a Teacher in Shufu High School (China) from 2008 to 2011. He received M.Sc. degree from Politecnico di Torino in March 2014. He worked as an Embedded Systems Engineer in ST Microelectronics from June to December 2014. Then he worked as a Software Developer in National Research Council of Italy from February 2015 to February 2016. Currently he is a Doctoral Assistant in the department of Electronics and Telecommunications of Politecnico di Torino under the supervision of Danilo Demarchi and Sandro Carrara. His main research interests include embedded intelligent system design for Bio-medical applications, analog front-end design for bio-sensors, machine-learning based algorithm development for sensor data processing and cloud based software development for healthcare.

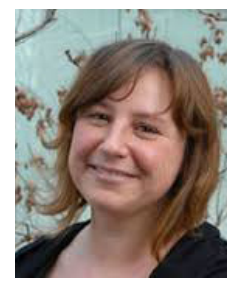

Séverine Lorrain received a Ph.D degree in plant science from the University of Toulouse (France) for work centred on the molecular pathways deregulated in an Arabidopsis lesion mimic mutants, in 2004. She then moved at the University of Lausanne in the group of Prof. Christian Fankhauser to decipher the molecular pathways involved on the plant responses to their changing light environment. From 2016, she is in charge of the Reverse Phase Protein Array platform at the Protein Analysis Facility of the University of Lausanne.

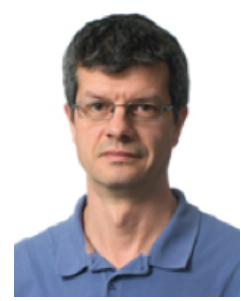

Manfredo Quadroni graduated in Biochemistry at the ETH Zurich and then received his Ph.D. from the same institution for work centred on calciummediated signalling and protein phosphorylation. There he also got his training in proteomics in the laboratory of Dr. Peter James. During a postdoctoral stay at the University of British Columbia, Canada, he also became familiar with signal transduction in immunology. A second post-doc brought him back to ETH Zurich, where he continued working on the development of methods for proteome analysis. He joined the University of Lausanne in February 2000 and has been in 
charge of the setup and operation of the Protein Analysis Facility of the same institution since 2002.

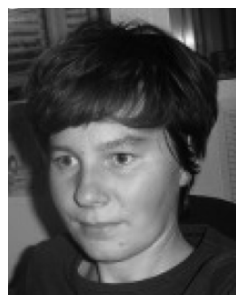

Marie-Agnès Doucey received a Ph.D. in biochemistry from the Friedrich-Miescher Institute at Novartis, Basel (Switzerland). She also holds a degree in Engineer in Biotechnology received at the Ecole Supéerieure de Biotechnologies de Strasbourg (E.S.B.S.), France. She is currently a project leader at the Ludwig Institute for Cancer Research at the University of Lausanne. Her current work focuses on the tumor microenvironment and the role of monocytes in breast cancer tumour development. She has more than twenty pubblications in peer-reviewed international journals.

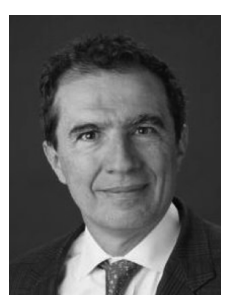

Giovanni De Micheli is Professor and Director of the Institute of Electrical Engineering at EPFL, Lausanne, Switzerland. Prof. De Micheli is a Fellow of ACM and IEEE, a member of the Academia Europaea and an International Honorary member of the American Academy of Arts and Sciences. His research interests include several aspects of design technologies for integrated circuits and systems, such as synthesis for emerging technologies, networks on chips and 3D integration. His citation h-index is 93 according to Google Scholar. He is member of the Scientific Advisory Board of IMEC (Leuven, B), CfAED (Dresden, D) and STMicroelectronics. Prof. De Micheli is the recipient of the 2016 IEEE/CS Harry Goode award for seminal contributions to design and design tools of Networks on Chips, the 2016 EDAA Lifetime Achievement Award, the 2012 IEEE/CAS Mac Van Valkenburg award for contributions to theory, practice and experimentation in design methods and tools and the 2003 IEEE Emanuel Piore Award for contributions to computer-aided synthesis of digital systems. He received also the D. Pederson Award for the best paper on the IEEE Transactions on CAD in 2018 and 1987, as well as several Best Paper Awards. He has been serving IEEE in several capacities, namely: Division 1 Director (2008-9), cofounder and President Elect of the IEEE Council on EDA (2005-7), President of the IEEE CAS Society (2003), Editor in Chief of the IEEE Transactions on CAD/ICAS (1997-2001). He has been Chair of several conferences, including Memocode (2014) DATE (2010), pHealth (2006), VLSI SOC (2006), DAC (2000) and ICCD (1989).

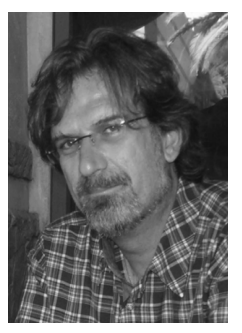

Danilo Demarchi (M'10-SM'13) received the master's and Ph.D. degrees in electronics engineering from the Politecnico di Torino (Polito), Italy, in 1991 and 1995, respectively. He is an Associate Professor with Polito and he is leading the Micro and Nano Electronic Systems Group, Department of Electronics and Telecommunications. He is also coordinating the microelectronics research line at the Center for Space Human Robotics, Istituto Italiano di
Tecnologia, Polito. He is author and co-author of two patents and over 150 scientific publications.

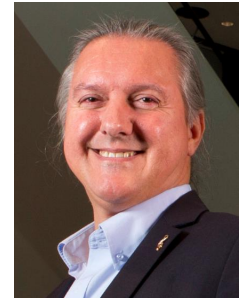

Sandro Carrara is an IEEE Fellow and also the recipient of the IEEE Sensors Council Technical Achievement Award. $\mathrm{He}$ is faculty at the EPFL in Lausanne (Switzerland), former professor at the Universities of Genoa and Bologna (Italy). He holds a $\mathrm{PhD}$ in Biochemistry and Biophysics, a Master degree in Physics, and a diploma in Electronics. His scientific interests are on electrical phenomena of nano-biostructured films, and include CMOS design of biochips based on proteins and DNA. Along his carrier, he published 7 books, one as author with Springer on Bio/CMOS interfaces and, more recently, a Handbook of Bioelectronics with Cambridge University Press. He has more than 250 scientific publications and is author of 13 patents. He is now Editor-in-Chief of the IEEE Sensors Journal, founder and Editor-in-Chief of the journal BioNanoScience by Springer, and Associate Editor of IEEE Transactions on Biomedical Circuits and Systems. He is a member of the IEEE Sensors Council, and was a member of the Board of Governors (BoG) of the IEEE CAS Society. He has been appointed two times as IEEE Distinguished Lecturer. His work received several international recognitions as bestcited papers and best conference papers. He has been the General Chairman of the Conference IEEE BioCAS 2014. 